\title{
Performance Based Design
}

\section{INTRODUCTION}

Michalatos and Kaijima underline in their paper 'Structural Information as Material for Design' (2007) the importance to approach an informed design consistency and respect the "criteria of efficiency, architectural intentions as well as intrinsic properties of the geometry" rather than simple structural optimization of a certain design. Our paper investigates this consistency between architecture and structure within the framework of parametric modeling, which requires architects, engineers and constructors to re-evaluate the feedback loop between how things are designed and constructed.

As Mario Carpo (2008) points out CAD and CAM technologies have overthrown the "Albertian Paradigm" which claims that architects should not make things, but should just design and annotate them. As digital tools can be used to design and fabricate at the same time, CAD-CAM technologies have already started to bridge the gap between designers and makers. One of the most influential formrelated factors on the lighting situation inside the building is - due to its shading behaviour - the dimension and position of the supporting structure of the façade.
It is important to investigate these positions at the beginning in depth, since they serve as hypothesis for the entire planning process. In order to compare a catalogue of various design approaches and different designs in a timely manner, a parametric model has been built defining the rough form of the design.

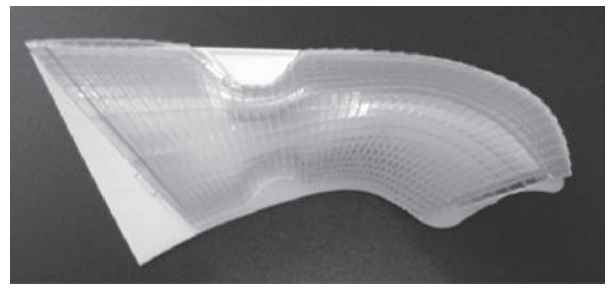

Figure 1: model greenhouse project

\subsection{THE GREENHOUSE PROJECT}

The project which is used for the described approach is a Greenhouse in Meran, Italy, with approximately $370 \mathrm{~m}^{2}$ gross area. The building has two programmatic parts: one smaller exhibition space and a big exhibition hall. These two spaces are separated by technical rooms. The transition between the two main spaces is visible in the outer shell of the building: here the shell tapers and emphasizes the transition 
zone. The section of the two exhibition halls varies. The arch system which is used for both exhibition halls has a maximum height of $10 \mathrm{~m}$ in the area of the big exhibition space and is 3 $\mathrm{m}$ lower in the area of the small hall. Therefore the arch system is more pointed in the big exhibition space than in the small. Towards the South the arch system rests directly on the concrete slab, whereas towards the North the arch system is lifted up by a $3 \mathrm{~m}$ high concrete wall. Next to the structural system the second main feature of the project is a faceted skin which uses rectangular planar panels to achieve the double-curved geometry.

\subsubsection{STRUCTURAL MODEL}

All parameters of the NURBS based geometry need to be editable and accessible in the architectural design software and the structural calculation software. The initial design development follows a constant loop between texture, structure and massing - each form study undergoes the following automated definition of the geometric parameters.

\section{1. parametrization of the structural calculation model.}

2. evaluating the structure versus light conditions.

3. looping back to 1 .

The result of this parametrization has been a rectangular grid of beams lying on a NURBS surface with editable grid densities, which is applicable to any given form. The structural calculation model contains not only geometry, but also loads resulting from various sources. Some of the loads directly result from the geometry, such as the weight of the structure itself. Others are indirectly related such as the weight of the glass panels, which depends on the grid size. As a consequence all changes in the geometry result in changes in the applied loads, the load distribution and lead therefore to a completely different model. In order to automatically create calculation models, loads must be realistically calculated and applied to the structure on the basis of algorithms. Additionally, further geometric information such as the element's profile orientation has to be calculated. A computer program using the .Net interface has been selfdeveloped to connect the CAD software and the calculation software. The final calculation models do not cover all loads and load combinations, such as wind loads, thermal expansion or imperfections, which had to be incorporated manually. As a result a set of different grids is structurally evaluated giving visual feedback to the dimension of the structural elements. Therefore it has beenpossible to compare the aesthetic of the design on the basis of realistic dimensions, while analyzing also the lighting requirements.

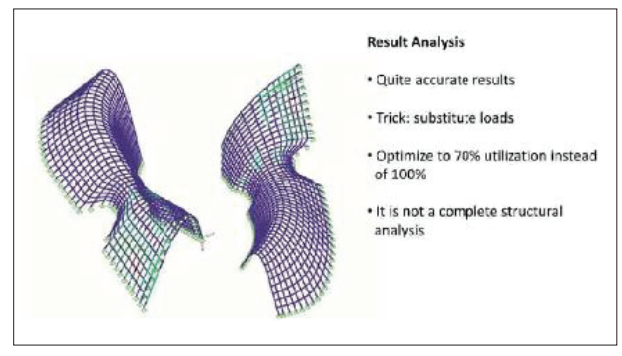

Figure 2: stress fields of the steel construction

\subsubsection{DEFINITION OF GEOMETRY MODEL PARAMETERS}

To be able to compare different designs in a timely manner, a parametric Grasshopper (McNeel Rhinoceros) model has been built, which defines the basic logic behind the structure of the given form. The final structure consists of flat steel elements aligned in a quadrangle grid. The grid consists of two different kinds of beam types: The primary structural beams, which span in the short direction and the secondary structural beams, which form a linear connection between two adjacent primary beams at several points. The secondary members correspond in size with the glass element, while the beam sections of the primary structural beams and the secondary structural beams may vary. To keep an even appearance of the structure the beam sections do not differ within the beam category. 


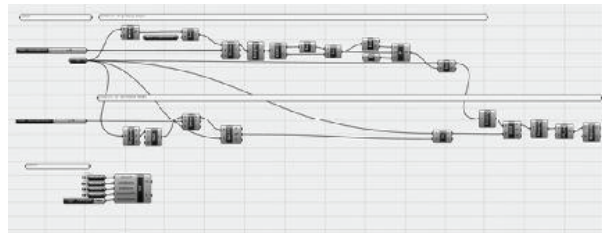

Figure 3: Grasshopper definition

The steel element sizes, the grid density and the resulting glass panel sizes have been explored under consideration of load bearing capacity and the resulting lighting condition.

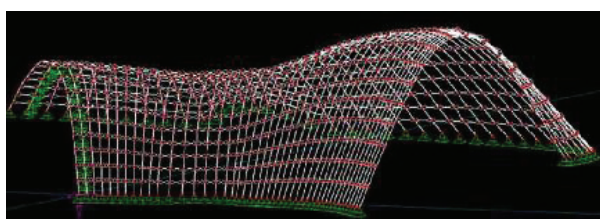

Figure 4: grid lines based on nurbs surfaces

The parametric model has been based on a NURBS Surface, representing the outer shell of the building. Onto the surface a rectangular grid of curves has been applied, which represent the axes of the beams. The main beam curves are defined by dividing two opposing surface edges into a parameter controlled number of elements, resulting in evenly spaced division points. Through two opposing division points a plane oriented in $\mathrm{z}$ direction has been created and intersected with the NURBS Surface creating the main beam curves. The location of the secondary structural beams is defined by ISO-Curves in V Direction of the surface using parameters resulting from equal division of one surface.

\subsubsection{ADDITIONAL DATA EXTRACTION}

The parametric grasshopper model is used as a base to create a structural model within the calculation software R-Stab (Dlubal). In our case the calculation software only interprets nodes and lines between these nodes, representing section beams. Nonetheless information bases on surfaces would be very useful to the program. In our approach the section rotation degree has been calculated on the base of the surface and applied to the subordinate structural beams within the structural model.

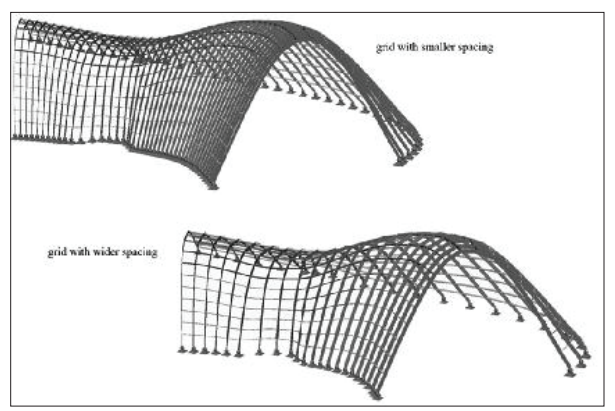

Figure 5: Calculation model with different grid densities

Other non-static, geometric properties have been defined to be interpreted differently in the calculation model. For example the start points and end points of the main beam curves are defined as hinged supports as well as the connection between the main and the subordinate structure is defined as a hinged connection. Next to the geometric information, loads resulting from geometric properties are reinterpreted and applied to the structural model. This approach has been used to calculate the distributed load of the panel weight and the snow load.

\subsubsection{AUTOMATED LOAD CALCULATION}

The structural model which derives from the parametric model has to be able to calculate the load cases by the parameter definition. The load cases which have been automatically created are:

1. own weight

2. distributed load of the panel weight

3. snow load

In addition to the automated load cases, load case combinations were automatically generated under consideration of the German standards (DIN 18800) and second order theory. The own weight of the structure has been au- 
tomatically calculated by the structural calculation program and only requires the sections as input. The weight is an important factor, because when changing the element section sizes, it significantly can change the outcome of the calculation. The distributed load of the panels though depends on the size of the panels and therefore has to be calculated for each parameter set separately. Based on each beam's allocated load area intersection points have been calculated which results, multiplied with a predefined area load of the panel weight, in the distributed load. The calculation of the allocated load area though is not a trivial problem. In order to calculate the area for an intersection point in a quadrangle grid one need to know the four closest points in all 4 directions and their relative position to the point in order to connect them. There is the need to implement a sorting algorithm based on the geometry. The first step has been to sort the unsorted list of beams and store the adjacent beams for each beam, by evaluating the $U$ value of each start point and connecting it with the beam object. So the curves with the next higher and lower $U$ value are the ones lying next to the main curve. All division points in the grid lie on a main beam curve and have different $t$-values. Their relative linear position is therefore defined and is separately stored in an index manner corresponding with the point location on the curve. Through the index of each point it is possible to get the next point $(i=i+1)$, the previous point $(i=i-1)$ and the points on the left and right with the same index on the left or right neighbor curve. By knowing these four points, which all lie on the base surface, it is possible to get the UV

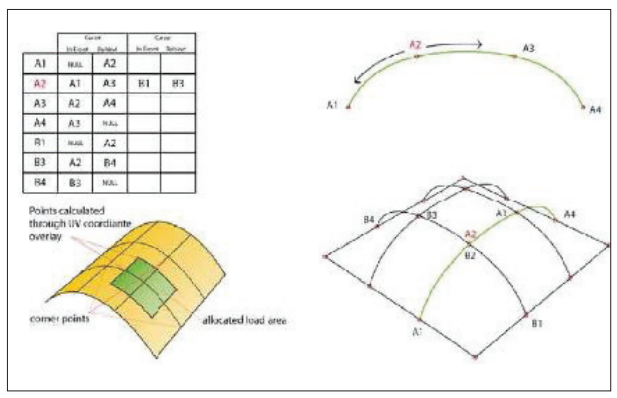

Figure 6: load area diagram coordinates of the points and create the new four corner points of the load area by overlaying the coordinates. The interpolated lines on the surface connecting the corner points define the edges of the load area surface.

To calculate the load area for snow load the surfaces simply need to be projected to the $x y$ plane. This approach does not consider snow sliding down the sides of the roof.

\subsection{WORKFLOW}

After the creation of the parametric model and the setup of the interface to the structural calculation program a process had been manually triggered, which creates a structural calculation model on the basis of the current parameter set. The calculation model then is automatically calculated. In an iterative process the section dimension is optimized to the applied loads. The result is saved in the native file format of the calculation program, giving a feedback to the dimension of the structural elements. With this realistic estimate of the structural dimensions a visual evaluation of the lighting requirements is possible.

\subsubsection{ERROR AVOIDANCE}

While automatically creating structural calculation models, it is very important to avoid calculation errors. A calculation error produced by non diverging load combinations in later iterations leads to a back step in the section size.

\subsubsection{QUALITY OF THE CALCULATED RESULTS}

The created calculation models do not cover all loads and load combinations, such as wind loads, thermal expansion or imperfections. Nonetheless the resulting section dimension is very realistic. The section optimization process does not optimize the elements to the highest degree of stress utilization of 100 percent, but a maximum utilization of 70 percent. Therefore there still is a buffer of 30 percent, which is very 
unlikely to be exceeded. However the result is not a complete structural analysis and additional load cases and load combinations would have to be added to reach the final state. The automatically created model though can be used as the basis for the final model. That alone is a great advantage in comparison to a nonautomated approach, since the modeling of a structural calculation model is a time consuming procedure, with a significant percentage of the overall editing time.

\subsection{ROUGHENING OF THE SKIN SYSTEM}

The structural system was has been used as a starting point for the modeling of the glass panels. Usually tessellation is a phenomenon that architects and engineers try to prevent. In case of the glasshouse an early decision was made that the tessellation of the skin needs a lot of intention. During the process a solution has been found which does not hide the tessellation, but places additional emphasize on the facets of the skin. A script has been written which always affected four points at a time. First a plane has been created through the first three points and has been rotated outwards in direction of surface normal in order to increase the discontinuity between the adjacent panels. Afterwards all four points were projected onto that plane, so that all four points meet in the same plane. This method ensures planar panels and creates an additional roughness of the panels.

The jaggedness of the skin is also affecting the main structural beams which now need to accommodate the jagged profile on the outside, whereas they can remain smooth on the inside. It is proposed to fabricate the main beams using water jet cutting technology. The jagged main beams also allow attaching the glass directly to the structure. The insulated glass layer consists of laminated safety glass on the inside and toughened safety glass on the outside. The glass panel is glued to a support bracket which is connected to the structure. The additional roughening of the façade allowed integrating a sun shading device on the outside. Each glass panel has its own shading element.

\subsection{CONCLUSION}

Through this integrative form finding process, a set of different grids was structurally evaluated giving feedback to the dimension of the structural elements. It was possible to compare the aesthetic of a design on the basis of realistic dimensions of the supporting structure and through that assure that the lighting requirements resulting from the structure are met. ${ }^{6}$

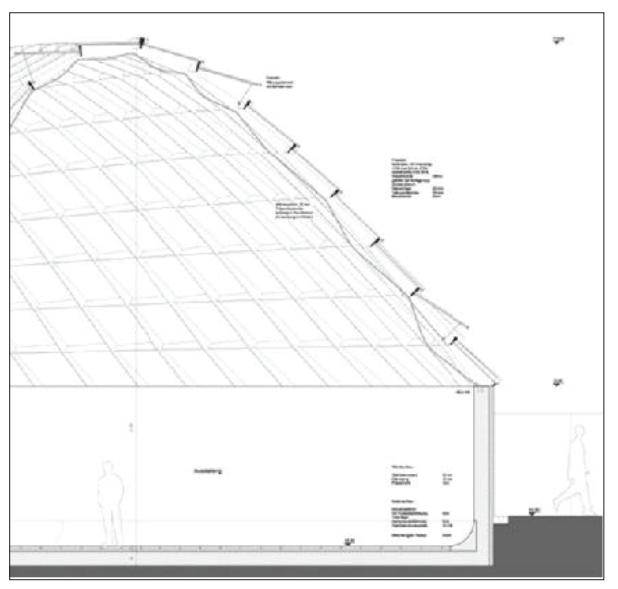

Figure 7: facade section

However the described workflow could be further extended in many ways. Especially the automated load calculation leaves room for further development. The goal would be to reduce the amount of manual editing time, for example by defining different wind load application areas on the surface to which a parameter set can refer to and automatically apply wind loads to the generated structure. Another promising approach would be to include illumination data as an additional numeric parameter by connecting an evaluation tool such as Ecotect to the developed software. Both of approaches mentioned above are currently investigated by us. 


\subsection{PROJECT 2}

The second project is based on two exhibition installations. The first installation was designed and built in $\mathbf{2 0 1 0}$ for the Designers Saturday in Langenthal, Switzerland - called the DS 2010 project from here on. The second installation is scheduled to be exhibited at the Passages in Cologne in January 2012- called the Passages 2012 project from here on.

The two projects are based on the same construction system that explores structure and ornament as one entity in an anti-Albertian approach. . The DS 2010 project will be discussed in so far as it informs Passages project 2012. The DS 2010 project focuses on tessellation to create aesthetic enjoyment, whereas the Passages 2012 project studies structural aspects: perceptive performance vs. structural performance.

\subsubsection{PROJECT OUTLINE}

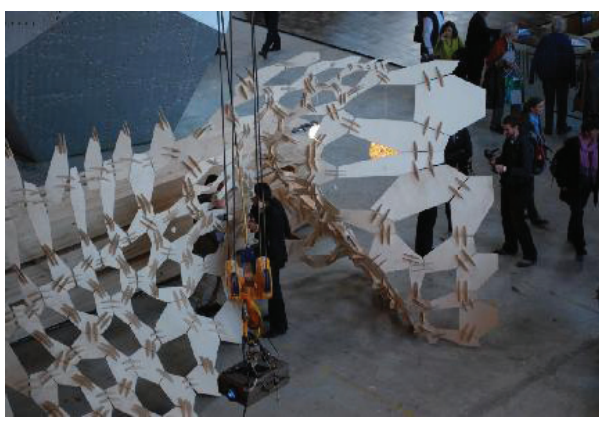

Figure 8. Exhibition at the DS2010 in Langenthal,

\section{Switzerland}

The DS installation is a wall that twists to create a passage through the installation. The project uses a surface tessellation that is directly reacting to surface curvature. The polygonal tessellation transforms in order to react to changes in curvature by scaling the size of the module and by reconfiguring the typology (notes within the polygonal structure vary through their local relations).

The surface was designed to create a varying tessellation. Flat areas are tessellated with relative large panels, whereas highly curvi-linear areas require smaller panels to follow the curvature. Folds are also introduced as a way of stiffening the surface. The installation is constructed out of plywood with a Stecksystem. All elements are different and unique. Therefore one of the main challenges was to automate the numbering of the parts so that the wall could be assembled easily. The installation was fabricated through waterjet technology, even though wood is not ideal for waterjet cutting. The machine had to be modified to use as little water as possible to void damage to the plywood, which was successful.

For the first project no structural calculations were made due to a narrow time frame for design and fabrication. The structure had to be tested and corrected during the erection of the structure. Certain problems were not foreseen and had to be fixed through suspension cables.

\subsubsection{CONCLUSION}

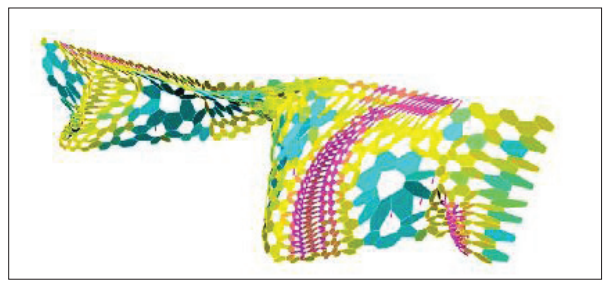

Figure 9: Size analysis DS 2010

The tessellation with varying parts was able to create an interesting aesthetic experience, but had deficits in structural and constructive performance. The first problem was resulting form parts that were too small. Small parts (pink) were weaker than medium scale and large scale parts because the small size of the panels only allowed for a small connection element, which weakened the structure substantially. The second problem was based on the decision to introduce a large variety of panel shapes from equilateral panels to elongated panels. The elongated panels created more tension in the connection pieces, which caused a few connections to fail. 


\subsection{PASSAGES 2011}

\subsubsection{DESIGN METHODOLOGY}

In order to avoid problems of the same sort the second exhibitions at the Passages 2011 tries to integrate structural design and spatial design at the same time.

Therefore a link between architects and engineers had to be defined at the beginning. This link is described in the following, even though the project might still change.

The exhibition proposal is also different formally. Instead of a wall the second project proposes a structure of arches that join in a cone.

Fig9 shows a series of optimizations. The model on the left shows the first tessellation. The tessellation follows a Nurbs surface, but the deviation form the surface curvature is not controlling the tessellation. The tessellation follows a structured grid. The panels have almost the same size: pink indicates small panels and blue large panels. But there are still two problems. Firstly the skin appears quite facetted in some areas. Secondly the blue panels that surround the cone tend to be elongated panels. The size of the pink of panels is already big enough to avoid the problem of weak connections.

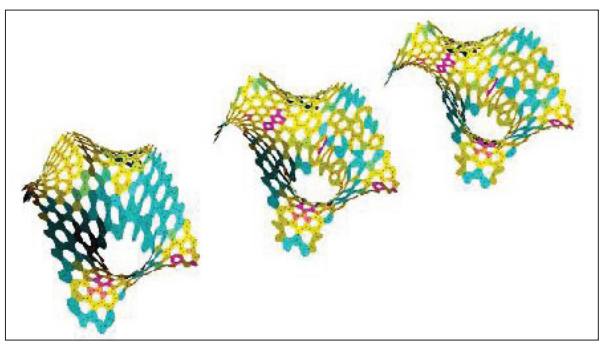

Figure 10: Optimization of the structural system from left to right

The next iteration, diagram in the middle, shows the model after a surface relaxation procedure: in general the surface is 8 smoother and we can observe that the relaxation algorithm has produced more equilateral panels.
The last iteration tries to create even more equilateral panels.

\subsubsection{STRUCTURAL MODEL}

In the design process exchanging structural data between architects and engineer became essential. The architects prepared a model that contained the centroids, the midpoints of each polygon edge that was connecting to another panel and lines that were connecting these points.

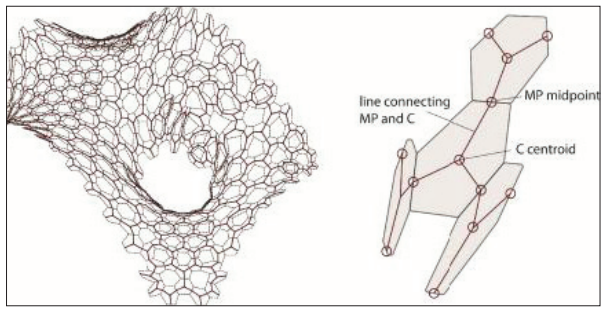

Figure 11: Geometric information that was transferred from Rhinoceros to RStab

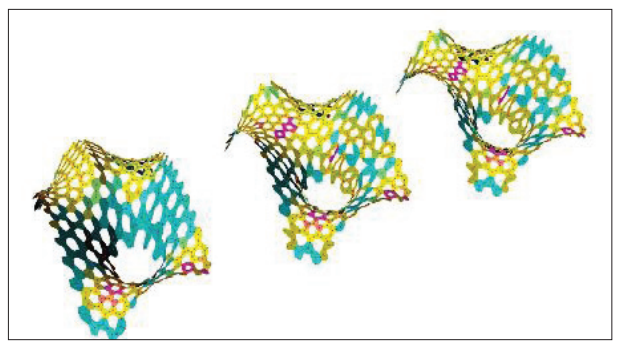

Figure 12: Variation of the module

Currently we are studying ways to modify the module in such a way that each module is changing according to its structural performance: the module thickens where it necessary and gets thin in areas with lower structural performance. This we can achieve varying the outer perimeter of the hexagonal panel and changing the inner opening of the panel. In order to automate this process a link between Grasshopper and R-Stab has been established to study the results in real time. Angles analysis is automated so that feedback can be given almost in real time. 


\begin{tabular}{|c|c|c|c|c|}
\hline & & $\mathrm{x}$ & $y$ & $z$ \\
\hline 0 & cartesian & 14,9005209 & $\begin{array}{l}- \\
12,1236693\end{array}$ & $\begin{array}{l}- \\
82,6360747\end{array}$ \\
\hline 0 & cartesian & 14,748 & $\begin{array}{l}- \\
12,0749345\end{array}$ & $\begin{array}{l}- \\
82,5028191\end{array}$ \\
\hline 0 & cartesian & 14,976552 & $\begin{array}{l}- \\
12,2040763 \\
\end{array}$ & $\begin{array}{l}- \\
82,5590248\end{array}$ \\
\hline 0 & cartesian & 14,976552 & $\begin{array}{l}- \\
12,0919743\end{array}$ & $-82,846405$ \\
\hline 0 & cartesian & 15,0410959 & $\begin{array}{l}- \\
12,3468266 \\
\end{array}$ & $\begin{array}{l}- \\
82,4445008 \\
\end{array}$ \\
\hline 0 & cartesian & 15,1874228 & $\begin{array}{l}- \\
12,4267244\end{array}$ & \begin{tabular}{|l|}
- \\
82,5590248 \\
\end{tabular} \\
\hline 0 & cartesian & 14,9593096 & $\begin{array}{l}- \\
12,4096847\end{array}$ & 82,2154388 \\
\hline 0 & cartesian & 15,3270166 & $\begin{array}{l}- \\
12,4997183\end{array}$ & 82,6778612 \\
\hline 0 & cartesian & 15,4054365 & 12,4334078 & 82,9090805 \\
\hline 0 & cartesian & 15,3881941 & \begin{tabular}{|l|}
- \\
12,6390162 \\
\end{tabular} & $\begin{array}{l} \\
82,5654945 \\
\end{array}$ \\
\hline 0 & cartesian & 15,4541689 & $\begin{array}{l}- \\
12,7872974 \\
\end{array}$ & 82,4488141 \\
\hline
\end{tabular}

Figure 14: Automated angle analysis

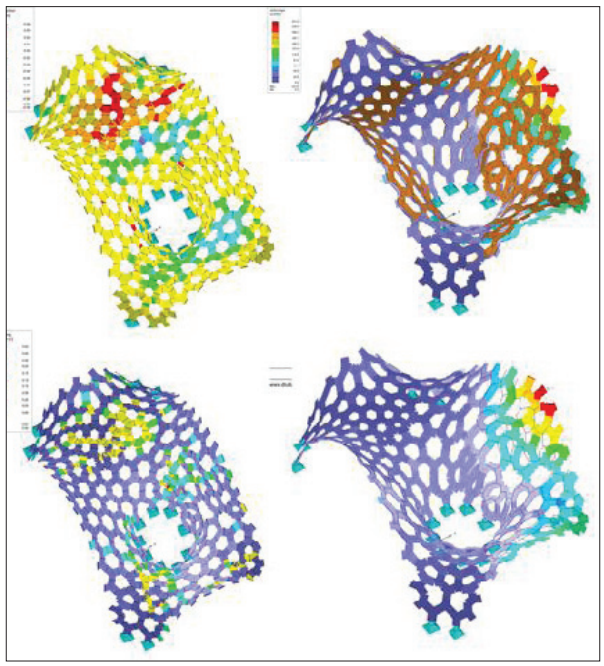

Figure 15. stress ratio and bending moment

\subsubsection{FABRICATION AND DETAILING}

We are currently building mock-ups with different connection details and also we are looking at alternative materials to plywood. But it seems likely that we will use the same material, but use cnc-milling instead of water jet cutting this time.

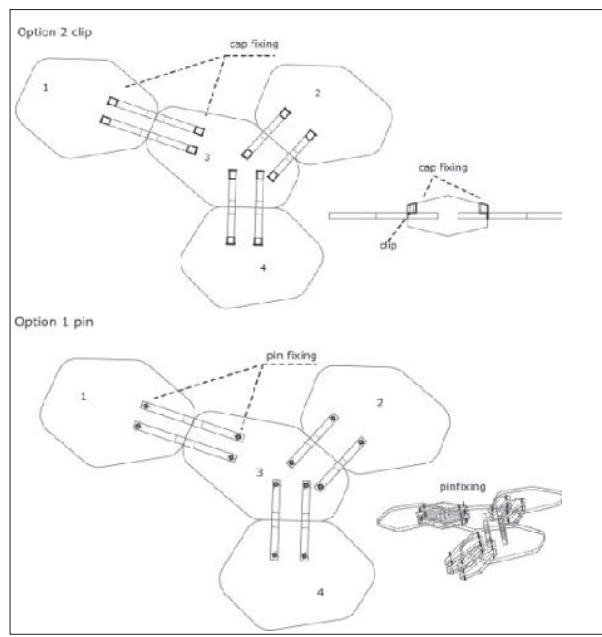

Fig16. Connection details

\section{OUTLOOK}

We see a high potential to introduce tools that facilitate collaboration between architects and engineers in an early stage for future projects. We think that the 19th century split between engineers and architects can be re-thought through collaborative tools that allow twodirectional feedback.

\section{REFERENCES}

Carpo, M: "Monstrous Objects, Morphing Things" In Monster, edited by Jacob Reidel et al. Perspecta 40 (2008) 16-21.

Carpo, M: 2008,"Non Standard Morality: Digital technology and its discontents" In: Architecture between Spectacle and Use, edited by Anthony Vidler (New Haven, CT: Yale University Press 2008) 127-142

Manegold, M.: "design tools for structural analysis early design stage", thesis at the university of Karlsruhe (2008)

Michalatos, Panagiotis and Kaijima, Sawako: Structural Information as Material for Design, Expanding Bodies: Art • Cities - Environment [Proceedings of the 27th Annual Conference of the Association for Computer Aided Design in Architecture, Halifax (2007) 84-95

Tessmann, Oliver: Structural Analysis as Driver in Surfacebased Design, Session 03: Collaborative Design and Presentation - eCAADe 25

Turrin, M. and Kilian, A. and Stouffs, R. and Sariyildiz: "digital design exploration of structural morphologies integrating adaptable modules", in: Tidafi,T. and Dorta,T.: Joining languages, cultures and visions: CAAD Futures (2009) 